\title{
CONSTRUCTION AND APPLICATION OF ENHANCED REMOTE SENSING ECOLOGICAL INDEX
}

\author{
Xinghan Wang ${ }^{1}$ *, Chaoqun Liu ${ }^{1}$, Qinghua Fu ${ }^{1}$, Bin Yin ${ }^{1}$ \\ ${ }^{1}$ Pearl River Institute of Hydraulic Research, China -rsgiswxh@126.com
}

KEY WORDS: Enhanced Remote Sensing Ecological Index, Ecosystem, Ecological Environment Quality

\begin{abstract}
:
In order to monitor the change of regional ecological environment quality, this paper use MODIS and DMSP / OLS remote sensing data, from the production capacity, external disturbance changes and human socio-economic development of the three main factors affecting the quality of ecosystems, select the net primary productivity, vegetation index and light index, using the principal component analysis method to automatically determine the weight coefficient, construction of the formation of enhanced remote sensing ecological index, and the ecological environment quality of Hainan Island from 2001 to 2013 was monitored and analyzed. The enhanced remote sensing ecological index combines the effects of the natural environment and human activities on ecosystems, and according to the contribution of each principal component automatically determine the weight coefficient, avoid the design of the weight of the parameters caused by the calculation of the human error, which provides a new method for the operational operation of regional macro ecological environment quality monitoring. During the period from 2001 to 2013, the ecological environment quality of Hainan Island showed the characteristics of decend first and then rise, the ecological environment in 2005 was affected by severe natural disasters, and the quality of ecological environment dropped sharply. Compared with 2001, in 2013 about 20000 square kilometers regional ecological environmental quality has improved, about 8760 square kilometers regional ecological environment quality is relatively stable, about 5272 square kilometers regional ecological environment quality has decreased. On the whole, the quality of ecological environment in the study area is good, the frequent occurrence of natural disasters, on the quality of the ecological environment to a certain extent.
\end{abstract}

\section{INTRODUCTION}

Ecosystems are an important living environment for humans and other creatures on earth, population growth, development and utilization of natural resources, and rapid economic development all exert some pressure on the quality of regional ecological environment(Song and $\mathrm{Xu}$ 2016). Therefore, it is of great significance to strengthen the monitoring of ecological environment quality so that policymakers can understand the status quo of regional ecological environment and better guide the sustainable development of human society(Pauleit and Duhme 2000; Li et al. 2015). At present, there is no clear definition of the quality of ecological environment(Li et al. 2015), and the method of evaluating the quality of ecological environment lacks a unified normative standard. The evaluation of the environmental quality of ecosystems is mainly evaluated by constructing indicators that affect different aspects of the ecosystem environment. In 2006, the Ministry of Environmental Protection promulgated the Technical Specification for Ecoenvironmental Status Evaluation in the form of industry standards and put forward the Eco-Environmental Status Index. The evaluation index mainly adopts five single indexes of biological abundance, vegetation coverage, land degradation, environmental quality and water network density for weighted summation.However, in practical application, there are some limitations in the promotion such as difficulty in determining the weight coefficient, difficulty in obtaining some single indicators and inability to display the spatial and geographical location.

With the deepening of researches on remote sensing technology in the field of ecological environment, many scholars have studied the effects of river ecology(Lyche et al. 2013), land development on the ecological environment(Lorenz and Feld
2013; Gu et al. 2014), vegetation ecology(Sun et al. 2013) and urban heat island using remote sensing indexes(Santamouris 2014), unique advantages in ecological environment monitoring. Among them, based on the principal component analysis method, the ecological index of remote sensing is proposed from four aspects affecting the greenness, humidity, dryness and heat of the ecosystem(Xu 2013a; Xu 2013b), overcoming the shortcoming of the single indicator and the difficulty of determining the weight in the traditional method, this method has been applied to a certain extent. However, since the remote sensing ecological index is mainly applied to the land area, it is easy to affect the humidity component in a large area of the water, and the research shows that the normalized vegetation index is adapt to be saturated with the detection of the high vegetation coverage area(Huete et al. 2002). Therefore, according to different vegetation coverage the vegetation index be selected.The environmental quality of the ecosystem is mainly manifested in three aspects, they include production capacity, changes in external disturbances and the impact of human social and economic development. This study proposed three indicators of net primary productivity, vegetation index and light index, using the principal component analysis of the contribution of the main components, and then automatically determine the weight coefficient, the formation of enhanced remote sensing ecological index.

Based on this, this paper proposes a multi-factor coupling ecoenvironmental quality monitoring index based on remote sensing image analysis from the three main aspects affecting the quality of ecosystems. They include production capacity, external disturbances, and human social and economic development.

* Corresponding author 
Through this study provides a rapid and effective method for the monitoring of ecological environment quality.

\section{CONSTRUCTION ENHANCED REMOTE SENSING ECOLOGICAL INDEX}

\subsection{Fundamental}

According to the research of relevant scholars (Cheng et al. 2015), the environmental quality of the ecosystem mainly manifests itself in three aspects: production capacity, external disturbances, and human social and economic development. Based on this, this study proposes three indicators based on the net primary productivity, vegetation index and light index, using the principal component analysis of the contribution of each principal component, and then automatically determine the weight coefficient, and build an enhanced remote sensing ecological index. Among them, the net primary productivity represents the production capacity of the ecosystem, the vegetation index represents the change of the ecosystem from outside interference, and the light index represents the socio-economic development of human beings. Therefore, the constructed enhanced remote sensing ecological index can be expressed by the above three indicators, ie:

$$
\begin{aligned}
\text { ERSEI }=f(\mathrm{NPP}, \mathrm{VI}, \mathrm{LI}) \\
\mathrm{VI}=\left\{\begin{array}{l}
\text { NDVI: high vegetation } \\
\text { EVI: medium }- \text { low vegetation }
\end{array}\right.
\end{aligned}
$$

In the formula, NPP is the net primary productivity, VI is the vegetation index, LI is the light index, and when the study area is covered by high vegetation, the EVI index is selected. When the study area is covered by medium-low vegetation, the NDVI index is selected.

\subsection{Single Index Construction}

2.2.1 Net Primary Productivity: Net primary productivity defines the rate at which all plants in the ecosystem produce net useful chemical energy. It is the difference between the rate at which a plant produces useful chemical energy in an ecosystem and the rate at which it is used for breathing energy (Melillo et al. 1993). This indicator can reflect the production capacity of ecosystems and is widely used in research areas such as climate change(Yan et al. 2017; Nemani 1999), carbon cycle (Zhang et al. 2016) and ecosystem (Wang et al. 2017) changes. For the calculation model of net primary productivity, domestic and foreign scholars have conducted a series of studies. In this study, a remote sensing estimation model for primary vegetation productivity based on light energy utilization is used. The detailed calculation method is described in (Zhu et al. 2007).

2.2.2 Vegetation Index: Vegetation change is an important reflection parameter in the change of ecological environment (Detsch and Otte 2016). For the analysis of vegetation changes, the NDVI and EVI are commonly used in the world currently. Among them, studies have shown that NDVI is prone to vegetation saturation in high vegetation coverage areas (Huete et al. 2002). Therefore, it is necessary to select NDVI or EVI according to the conditions of different research areas. The calculation methods are formula (3) and formula (4).

$$
\begin{gathered}
\text { NDVI }=\frac{\text { band } 2-\text { band } 1}{\text { band } 2+\text { band } 1} \\
\text { EVI }=2.5 * \frac{\text { band } 2-\text { band } 1}{\text { band } 2+6 * \text { band } 1-7.5 * \text { band } 3+1}
\end{gathered}
$$

In the formula, band 1 represents the red band, band 2 represents the near-infrared band, and band 3 represents the blue band.

2.2.3 Light Index: The light index detects weak nighttime lighting information in the city, Thus indirectly reflecting the development of the city, it is also called the economic index. There is a unique advantage in the study of macroscopic human social and economic development (Elvidge et al. 1999; Liu and Zhou 2017). And it has been well applied in urban expansion monitoring, GDP, urbanization level, population estimation, and carbon emission estimation (Zhang et al. 2017). Therefore, in this study, the remote sensing light index is adopted as the embodiment of human economic and social development, which avoids screening and statistics of a large number of cumbersome social and economic indicators, and facilitates the operationalization of ecological environment monitoring.

\subsection{Comprehensive Index Construction}

In order to reflect the features of ecosystem environmental quality that are influenced by many factors, This study selects three indicators of the net primary productivity, vegetation index and light index from the two major aspects of the natural environment and human activities. The principal component analysis method is used to determine the weight coefficient, and an enhanced remote sensing ecological index is constructed. In order to facilitate the principal component analysis, the index components are first normalized. See equation (5) for the calculation method.

$$
\mathrm{M}=\frac{M_{i}-M_{\min }}{M_{\max }-M_{\min }}
$$

In the formula, $M$ represents the value of each cell after normalization. $\mathrm{M}_{\min }$ Represents the minimum value of all pixel values in the study area. $M_{\max }$ Represents the maximum value of all pixel values in the study area. $\mathrm{M}_{\mathrm{i}}$ Represents cell values at any location within the study area. Among them, the extraction and normalization calculation of the maximum pixel value and the minimum pixel value are all based on ENVI5.0 software platform processing.

Further, based on the single index after the completion of the normalization, the comprehensive index is constructed, see Formula (6).

$$
\text { ERSVI }=\eta * \mathrm{PC} 1+\lambda * \mathrm{PC} 2+\mu * \mathrm{PC} 3
$$

In the formula, $\eta, \lambda$ and $\mu$ are the weights of the eigenvalue contribution of the principal component analysis of the net primary productivity, vegetation index, and light index, respectively. The greater the ERSVI value, the better the ecological environment quality.

\section{STUDY AREA AND DATA}

This article uses Hainan Island as a research area. The data of net primary productivity, vegetation index, and light index from 2001 to 2013 were selected, and preprocessing of mosaic images, projection transformation, radiation correction, resampling, and other remote sensing images was performed. Among them, the net primary productivity is the MODIS satellite MOD17A3 dataset with a spatial resolution of $1 \mathrm{~km} \times 1 \mathrm{~km}$ and time resolution of year. The vegetation index uses MOD13A3 data from MODIS satellites with spatial resolution of $1 \mathrm{~km} \times 1 \mathrm{~km}$ and time resolution of months. By synthesizing monthly data, you can get data in years. Vegetation dataset includes NDVI and EVI vegetation 
index data. The light index uses the DMSP/OLS data set, spatial resolution is 30 arc seconds, resampled to $1 \mathrm{~km} \times 1 \mathrm{~km}$, the time resolution is years, and the range of data values is a grayscale value of $0-63$.

\section{RESULTS AND ANALYSIS}

Table 1 shows the results of principal component analysis of net primary productivity, vegetation index and light index for 2001, 2005, 2009 and 2013, Based on this, the enhanced remote sensing ecological index of the four years mentioned above is calculated in combination with formula (6), as shown in Figure 1.

\begin{tabular}{lcccccc}
\hline & \multicolumn{3}{c}{2001} & & \multicolumn{3}{c}{2005} \\
\cline { 2 - 7 } & PC1 & PC2 & PC3 & PC1 & PC2 & PC3 \\
Eigenvalues & 0.1567 & 0.0125 & 0.0047 & 0.1449 & 0.0126 & 0.0051 \\
contribution & $90.12 \%$ & $7.20 \%$ & $2.68 \%$ & $89.12 \%$ & $7.72 \%$ & $3.16 \%$ \\
\hline & & 2009 & & & 2013 & \\
\cline { 2 - 7 } & PC1 & PC2 & PC3 & PC1 & PC2 & PC3 \\
Eigenvalues & 0.1521 & 0.0127 & 0.0078 & 0.1618 & 0.0207 & 0.0108 \\
contribution & $88.08 \%$ & $7.39 \%$ & $4.53 \%$ & $83.69 \%$ & $10.73 \%$ & $5.58 \%$ \\
\hline
\end{tabular}

Table 1. Eigenvalues and contribution of three index
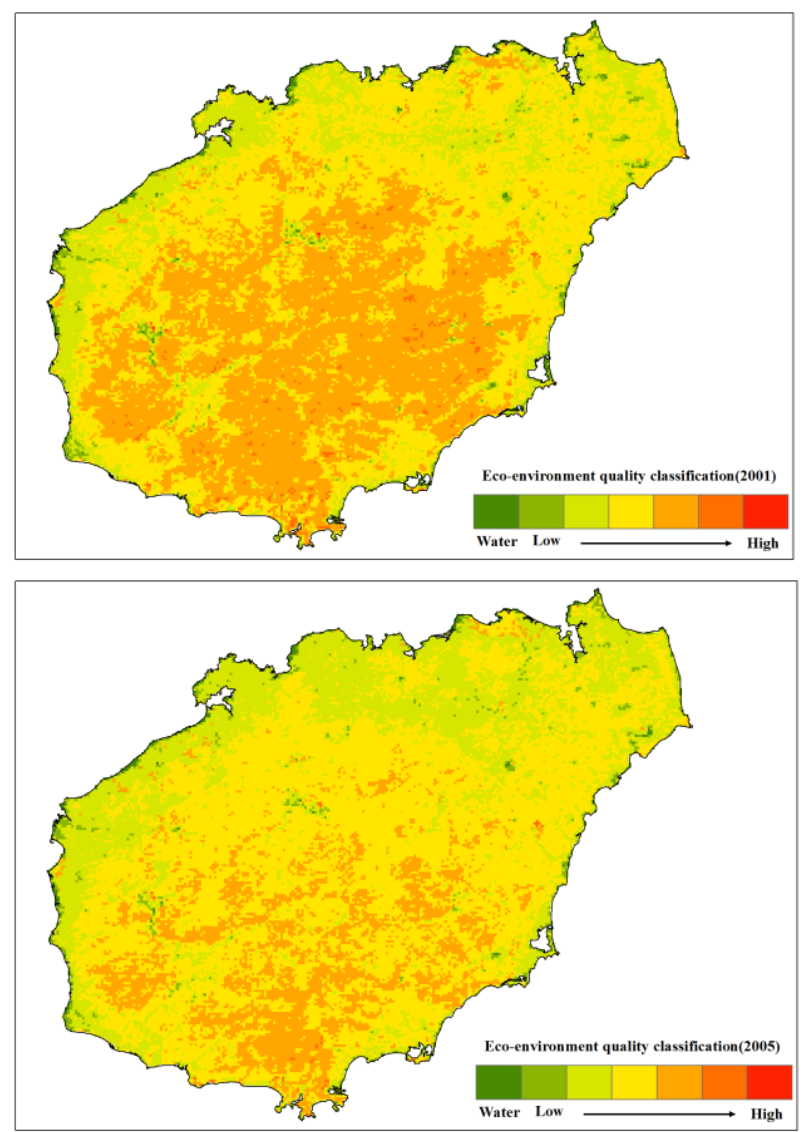
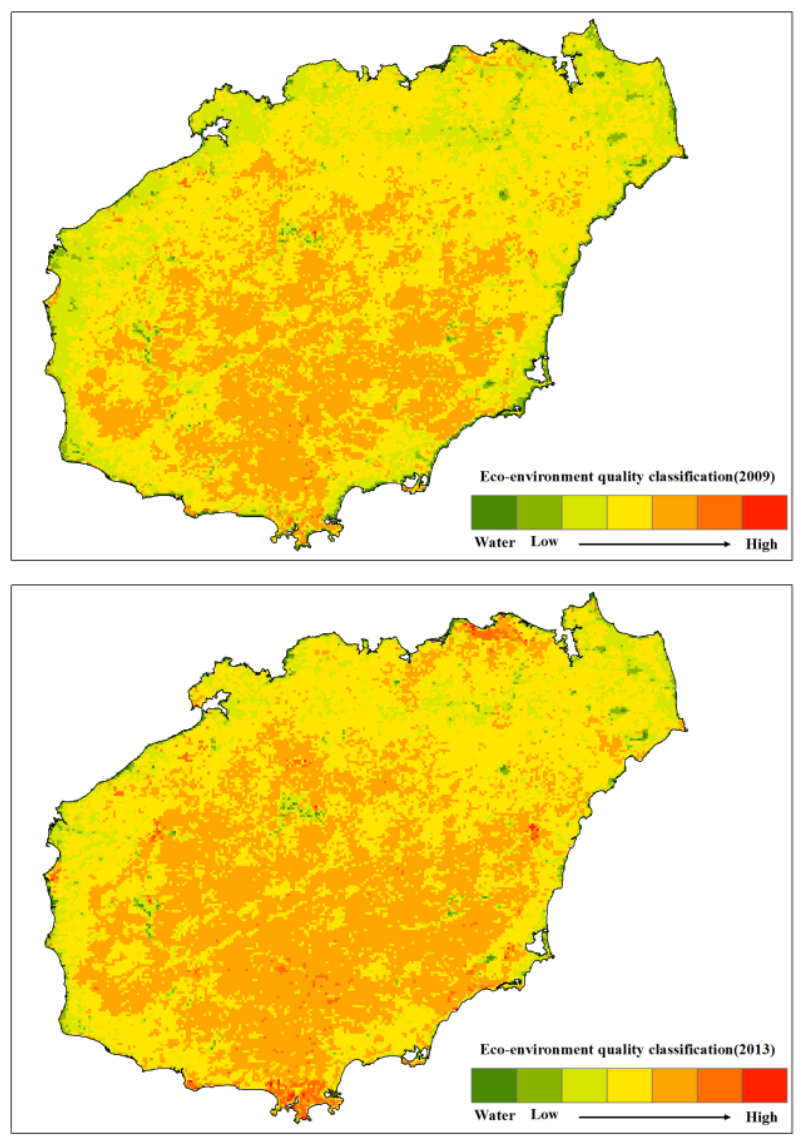

Figure1 The enhanced remote sensing ecological index images of study area

The results in Figure 1 indicate that during the period from 2001 to 2013, the ecological environment quality of Hainan Island experienced a process of first decreasing and then increasing. Using 2001 as the base year for comparison, in 2005, the quality of the ecological environment in Hainan Island decreased significantly. In particular, the changes in the central Baisha Li Autonomous County, Qiongzhong Lizu Miao Autonomous County, and Wuzhishan City of Hainan Island were significant. The main reason for the change was the major natural drought in Hainan Island during 2004 2005. As a result, the quality of the ecological environment of vegetation has dropped dramatically. As a result, there has been a marked decline in the quality of the ecological environment. After 4 years of natural ecosystem restoration, in 2009, the quality of the ecological environment was significantly improved and improved compared to 2005. As Hainan Island comprehensively promoted the construction of international tourism islands, the protection of the ecological environment was further strengthened. In 2013, the quality of the ecological environment was generally good. Statistics show that during 2001 2013, the ecological environment quality of the 20,000 square kilometers area in Hainan Island has been significantly improved. Mainly concentrated in the central area of Hainan Island. About 8760 square kilometers of regional ecological environment quality is relatively stable. The quality of the eco-environment in the area of about 5,272 square kilometers shows a downward trend. The overall ecological environment quality in the region is good. The occurrence of major natural disasters has caused a certain impact on the quality of the regional ecological environment. 


\section{CONCLUSIONS}

This study selected three major aspects affecting ecosystem quality. Selecting the net primary productivity represents the production capacity of the ecosystem, the vegetation index represents the change of the ecosystem by external disturbances, the light index represents the socio-economic development of human beings, and the principal component analysis method is used to construct an enhanced remote sensing ecological index. The index integrates the impact of the natural environment and human activities on the ecosystem. And the weight coefficient is automatically determined by the contribution of each principal component. Avoiding the artificial deviation of the calculation results, and provides new monitoring means for the operational monitoring of regional macro-environmental quality monitoring.

In this paper, we use the enhanced remote sensing ecological index to monitor the ecological environment quality of Hainan Island from 2001 to 2013. The results show that the overall quality of ecological environment in Hainan Island is good. The quality of the eco-environment shows the characteristics of changes that first fell and then rose. The occurrence of severe natural disasters has a certain impact on the quality of the ecological environment.

It is of great significance to carry out monitoring of the quality of the ecological environment for the sustainable development of the region. Different scholars have evaluated different aspects of the impact on ecosystems. However, there is no unified standard for the system of regional ecological environmental quality monitoring. The method studied in this paper integrates the two important factors of the natural environment and human activities, and the index factors are easy to obtain, and there are few human factors, which are suitable for popularization and application.

\section{ACKNOWLEDGEMENTS}

Research funded by the Natural Science Foundation of Guangdong project-titled "Estimation and Forecast of Suspended Sediment Concentration in the Pearl River Estuary from Remote Sensing Images based on Data Assimilation Algorithm" (2017A030313232), and Science and Technology Program of Guangzhou project-titled "Remote Sensing Identification and Dynamic Monitoring of Urban Black-Odor Water Bodies based on Chroma Parameters"(201804010270).The authors also wish to thank the anonymous reviewers for their valuable and useful suggestions that clearly improved this paper.

\section{REFERENCES}

Song, H. M., and Xue, L. 2016. Dynamic monitoring and analysis of ecological environment in Weinan city,Northwest China based on RESI model. Chinese Journal of Applied Ecology, 27(12),pp.3913-3919.

Pauleit S., Duhme F. 2000.Assessing the environmental performance of land cover types for urban planning. Landscape and urban planning, 52(1),pp.1-20.

Li, F. N., Chang, R. Q., Sheng, J., and Liu, J. 2015. Dynamic monitoring of ecological environment in loess hilly and gully region of Loess Plateau based on remote sensing. Chinese Journal of Applied Ecology, 26(12),pp. 3811-3817.
Lyche,S.A., Feld, C.K., Birk, S., et al. 2013. Ecological status assessment of European lakes: a comparison of metrics for phytoplankton, macrophytes, benthic invertebrates and fish. Hydrobiologia, 704(1), pp. 57-74.

Lorenz, A. W., Feld C. K. 2013. Upstream river morphology and riparian land use overrule local restoration effects on ecological status assessment. Hydrobiologia, 704(1), pp. 489-501.

Gu,K.K., Chu,J.L., and Wang,Y.Z.2014. Spatio-temporal analysis of land use and ecological carrying capacity in coal mining city based on remote sensing. Acta Ecologica Sinica, 34(20),pp. 5714-5720.

Sun,Y.G., Zhao,D.Z., Guo,W.Y., Gao,Y., Su,Y.,and Wei,B.Q. 2013. A review on the application of remote sensing in mangrove ecosystem monitoring. Acta Ecologica Sinica, 33(15),pp. 45234538 .

Santamouris M. 2014. Cooling the cities-a review of reflective and green roof mitigation technologies to fight heat island and improve comfort in urban environments. Solar Energy, 103, pp. 682-703.

Xu,H.Q. 2013a. A remote sensing urban ecological index and its application. Acta Ecologica Sinica, 33(24),pp. 7853-7862.

Xu,H.Q. 2013b. A remote sensing index for assessment of regional ecological changes. China Environment Science, 33(5),pp. 889-897.

Huete, A., Didan, K., Miura, T., et al. 2002. Overview of the radiometric and biophysical performance of the MODIS vegetation indices. Remote sensing of environment, 83(1), pp. 195-213.

Chen,Q., Chen, Y.H., Wang, M.J., Jiang, W. G., Hou, P., and $\mathrm{Li}$,Y. 2015. Ecosystem quality comprehensive evaluation and change analysis of Dongting Lake in 2001-2010 based on remote sensing. Acta Ecologica Sinica, 35(13),pp.4347-4356

Melillo, J. M., Mcguire, A. D., Kicklighter, D. W., et al. 1993. Global climate change and terrestrial net primary production. Nature, 363(6426), pp. 234-240.

Yan, C., Qirun, X., Xin, X., et al. 2017. Responses of Net Primary Productivity to Climatic Factors in Youyang County. Meteorological \& Environmental Research, , 8(1), pp. 21523940.

Nemani, R. R., Keeling, C. D., Hashimoto, H., et al. 2003. Climate-driven increases in global terrestrial net primary production from 1982 to 1999 . Science, 300(5625), pp. 15601563.

Zhang, Y., Xiao, X., Jin, C., et al. 2016. Consistency between sun-induced chlorophyll fluorescence and gross primary production of vegetation in North America. Remote Sensing of Environment, 183, pp. 154-169.

Wang, S., Zhang, B., Yang, Q., et al. 2017. Responses of net primary productivity to phenological dynamics in the Tibetan Plateau, China. Agricultural and Forest Meteorology, 232, pp. 235-246. 
Zhu, W., Pan, Y., Zhang, J. 2007. Estimation of net primary productivity of Chinese terrestrial vegetation based on remote sensing. Journal of Plant Ecology, 31(3), pp. 413-424.

Detsch F, Otte I, Appelhans T, et al. 2016. Seasonal and longterm vegetation dynamics from 1-km GIMMS-based NDVI time series at Mt. Kilimanjaro, Tanzania. Remote Sensing of Environment, 178, pp. 70-83.

Elvidge, C. D., Baugh, K. E., Dietz, J. B., et al. 1999. Radiance calibration of DMSP-OLS low-light imaging data of human settlements. Remote Sensing of Environment, 68(1), pp. 77-88.

Li, X., Zhou, Y. 2017. Urban mapping using DMSP/OLS stable night-time light: a review. International Journal of Remote Sensing, pp. 1-17.

Zhang, M., Miao, W., Yang, Y., et al. 2017. Spatial-Temporal Features of Wuhan Urban Agglomeration Regional Development Pattern-Based on DMSP/OLS Night Light Data. Journal of Building Construction and Planning Research, 5(01), pp. 14-29. 